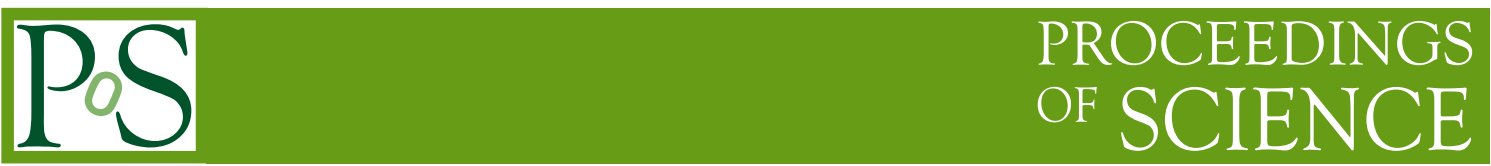

\title{
Cosmic reionization and the LOFAR project
}

\section{Benedetta Ciardi ${ }^{* *}$}

Max Planck Institute for Astrophysics

E-mail: ciardi@mpa-garching.mpg.de

In this talk I have presented theoretical models of the cosmic reionization process and discussed ongoing activities within the LOFAR Epoch of Reionization Working Group.

It has long been known that the most promising tool to investigate the EoR is via the $21 \mathrm{~cm}$ line from neutral hydrogen, which is expected to be seen in emission or absorption against the CMB. The generation of radio interferometers which is presently being built should be able to detect such line. Among these telescopes, LOFAR is in a very advanced construction phase and data are flowing. Thus, a great effort is being done within the Working Group to produce a number of ad hoc theoretical models to be compared with observations. The ultimate challenge for the detection of the cosmological signal though will be to separate it from the foreground signal, which is orders of magnitude higher. Thus, in addition to simulating mock reionization histories, an ongoing effort is being done to identify the best technique to extract the primary signal.

Cosmic Radiation Fields: Sources in the early Universe November 9-12, 2010

Desy, Germany

\footnotetext{
*Speaker.

${ }^{\dagger}$ Special thanks to all the collaborators mentioned in my talk.
} 


\section{Introduction}

After the Big Bang the Universe is filled with hot plasma. Following its expansion though the gas cools down and eventually becomes neutral at $z \sim 1300$ (recombination epoch). The period which follows is commonly known as the Dark Ages and ends when the first structures start to form and produce ionizing radiation. This marks the beginning of the reionization process, which is complete by $z \sim 6$. Eventually, these early structures evolve into those observed presently.

From an observational point of view, information on the Universe at the recombination epoch is available via the detection of the Cosmic Microwave Background (CMB) radiation, while the present generation of telescopes in the UV/optical/IR probe the cosmos up to $z \sim 8$ (e.g. Ota et al. 2007; Ouchi et al. 2009; Salvaterra et al. 2009). The new record has been set by a spectroscopically confirmed galaxy at $z \sim 8.6$ (Lehnert et al. 2010). Nevertheless a large observational gap still exist, that will be partially covered by the planned generation of instruments that will explore the FIR and radio bands.

Observational constraints on the reionization process. Because the intensity of the absorption observed in the quasars' spectra depends on the amount of HI present, observations of high- $z$ quasars are used to put constraints on the abundance of neutral hydrogen at high redshift. The value inferred from these observations though can vary substantially, with $x_{H I}>0.1, x_{H I}>0.033$, $x_{H I}<0.06$ (e.g. Wyithe \& Loeb 2004; Oh \& Furlanetto 2005; Mesinger \& Haiman 2007; Maselli et al. 2007; Bolton \& Haehnelt 2007), just to mention a few. Galaxies and GRBs are used as well as probes of the latest stages of the reionization process, as more and more are being discovered at redshift even higher than those of the quasars.

An additional observational constraint comes from the detection of anisotropies in the power spectrum of the $\mathrm{CMB}$, induced by the interaction between the CMB photons and the electrons produced during the reionization process. More precisely, the value of the Thomson scattering optical depth, $\tau_{e}$, measured by WMAP after $1,3,5$ and 7 years of operation is $0.16 \pm 0.04,0.09$ $\pm 0.03,0.087 \pm 0.017$ and $0.088 \pm 0.015$ respectively (Kogut et al. 2003; Spergel et al. 2007; Dunkley et al. 2008; Larson et al. 2010). As $\tau_{e} \propto \int n_{e}(z) d z$, where $n_{e}$ is the electron number density, it gives an estimate of the global amount of electrons produced during the reionization process, but no information is available on how these electrons are distributed in space and time.

Thus, the available observations offer constraints on the latest stages of reionization and on the global amount of electrons produced, but to have information on, e.g., the sources of ionization or the history of the reionization process, different type of observations are needed (see Sec. 3).

Theoretical modeling of the reionization process. The ingredients necessary to describe the reionization process are the following:

- a model to follow the formation and evolution of galaxies, possibly including the various feedback effects that regulate the process;

- the properties of the ionizing sources, which could be of stellar type, quasars or more exotic sources like e.g. dark matter decay or annihilation;

- a method to follow the propagation of the photons emitted by such sources. 


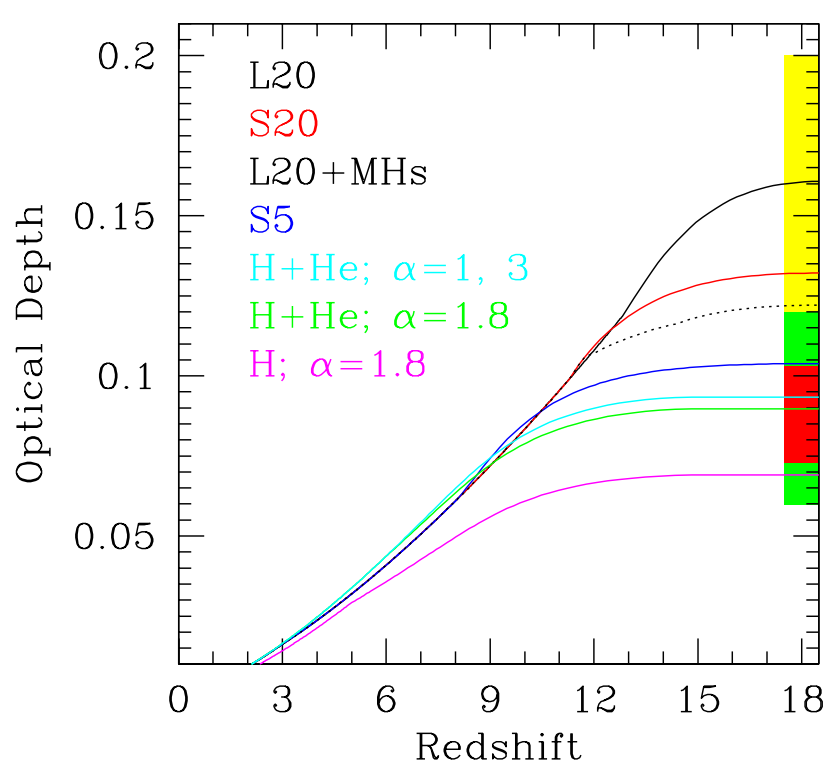

Figure 1: Evolution of the Thomson scattering optical depth for different simulations. Details can be found in Ciardi, Stoehr \& White (2003), Ciardi, Ferrara \& White (2003), Ciardi et al. (2006), Ciardi et al. in prep. The yellow, green and red rectangles refer to the yr-1, 3 and 5 WMAP measurements, while the yr-7 is very similar to yr-5 (see text).

While the second item is probably the most uncertain and parameters (e.g. for stellar type sources their Initial Mass Function [IMF] or the escape fraction of ionizing photons, $f_{e s c}$ ) are needed to describe the properties of the sources, the third item is the most challenging from a numerical point of view. In fact, the full solution of the seven dimension radiative transfer (RT) equation (three spatial coordinates, two angles, frequency and time) is still well beyond our computational capabilities and, although in some specific cases it is possible to reduce its dimensionality, for the reionization process no spatial symmetry can be invoked. Thus, an increasing effort has been devoted to the development of radiative transfer codes based on a variety of approaches and approximations, which have been tested and compared in Iliev et al. (2006).

\section{Simulations of cosmic reionization}

Through the years our group has run several simulations of IGM reionization (Ciardi, Stoehr \& White 2003: Ciardi, Ferrara \& White 2003; Ciardi et al. 2006) (i) using simulations of galaxy formation run with the Gadget code (Springel, Yoshida \& White 2001) to retrieve the properties of gas and galaxies; (ii) adopting different characteristics for the source properties (i.e. adopting for the stellar sources a standard Salpeter IMF or a Larson IMF biased towards massive stars; varying the value of the escape fraction of ionizing photons in the range 5-20\%; using quasar type sources); 
(iii) using the Monte Carlo radiative transfer code CRASH (Ciardi et al. 2001; Maselli, Ferrara \& Ciardi 2003; Maselli, Ciardi \& Kanekar 2009; Pierleoni, Maselli \& Ciardi 2009) to follow the propagation of ionizing photons. In some simulations we have also included the physics of $\mathrm{He}$ (Ciardi et al. in preparation). In this case we have adopted a range of small and medium size boxes (between 2 and $40 \mathrm{Mpc}$ comoving), the results of which are used to device a sub-grid model to run simulations with the Millennium Simulation.

The reionization histories mentioned above produce a Thomson scattering optical depth in the range 0.07-0.17, depending on the parameters/approximations adopted (see Fig.1). Thus, the uncertainties in the theoretical modeling of the reionization process are still large enough to accommodate the value of $\tau_{e}$ measured by the WMAP satellite (see Introduction). In order to put more severe constraints on our theoretical models a different type of observations is needed.

\section{3. $21 \mathrm{~cm}$ line diagnostic}

The ideal observation to shed light on the reionization process is the detection of the $21 \mathrm{~cm}$ line from neutral hydrogen (see Furlanetto, Oh \& Briggs 2006 for a review on the topic). The ground state of $\mathrm{HI}$ has a hyperfine structure and anytime a transition takes place between the hyperfine states a photon is emitted or absorbed with a wavelength of $21 \mathrm{~cm}$. Thus, observations at different frequencies would give information on the state of $\mathrm{HI}$ at different redshifts, providing insight on the spatial and temporal evolution of the reionization process.

Such a line is expected to be observed in terms of the effects that it has on a background radiation. Typically, the $\mathrm{CMB}$ is considered. What we expect to observe is then the differential brightness temperature, $\delta T_{b}$, between the CMB and a patch of HI with optical depth $\tau_{H I}$ and spin temperature $T_{S}$ (which regulates the population of the atomic levels). This is given by:

$$
\delta T_{b} \sim \frac{T_{s}-T_{C M B}}{1+z} \tau_{H I} \propto\left(1-\frac{T_{C M B}}{T_{s}}\right),
$$

where $T_{C M B}$ is the CMB temperature. From the above equation follows that if $T_{s}$ is equal to $T_{C M B}$ $\delta T_{b}$ is zero and no line can be observed, if instead it is larger (smaller) than $T_{C M B}$ the line is expected to be observed in emission (absorption) against the CMB. Thus, the value of the spin temperature is crucial for the observation of the line. $T_{s}$ can be written as a weighted mean between $T_{C M B}$ and the kinetic temperature of the gas, $T_{k}$ :

$$
T_{s}=\frac{T_{C M B}+\left(y_{\alpha}+y_{c}\right) T_{k}}{1+y_{\alpha}+y_{c}}
$$

where $y_{\alpha}$ and $y_{c}$ is the efficiency in coupling $T_{s}$ to $T_{k}$ due to Ly $\alpha$ scattering and collisions (with $\mathrm{H}$ atoms, electrons and protons) respectively (see Ciardi \& Salvaterra 2007 for the values adopted here). While at $z>20 y_{c}$ is dominant, at lower redshift scattering with the Ly $\alpha$ photons emitted by the first stars is the most efficient process (Wouthuysen 1952; Field 1959; Hirata 2006; Pritchard \& Furlanetto 2006). In addition, Ly $\alpha$ photons could be able to heat the IGM temperature above the CMB temperature and render the $21 \mathrm{~cm}$ line visible in emission.

The general picture that we expect is shown in Figure 2 for two different populations of stars producing Ly $\alpha$ photons, i.e. metal-free stars with a Salpeter IMF and Very Massive Stars (VMS) 


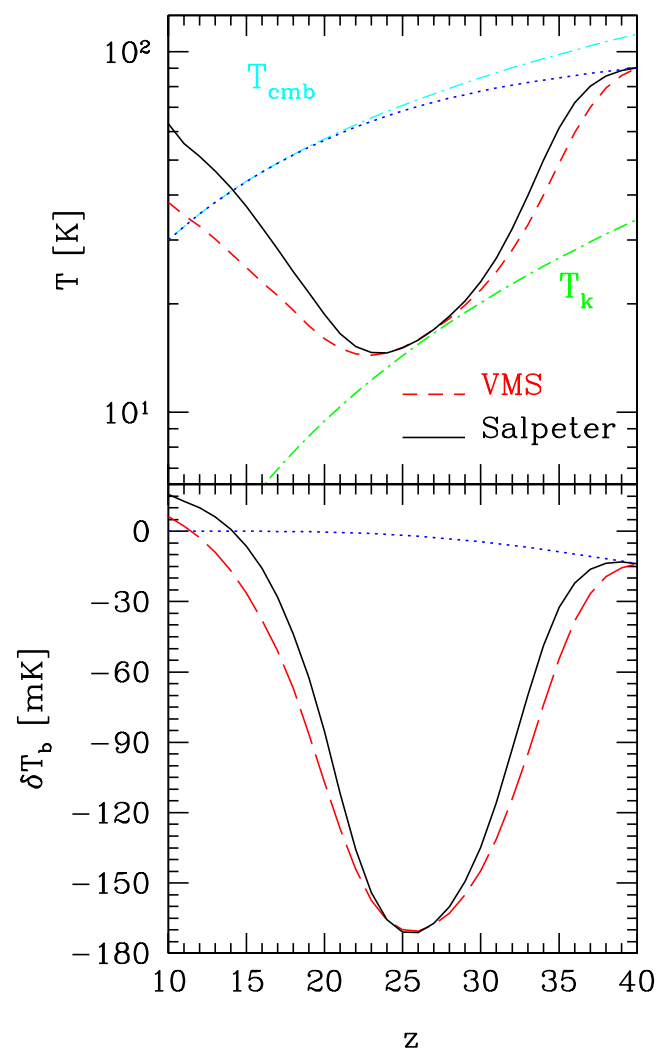

Figure 2: Upper panel: Evolution of the spin temperature for a Salpeter (solid lines) and a VMS (dashed) IMF, and in the absence of a Ly $\alpha$ background (dotted). $T_{C M B}$ and $T_{k}$ are plotted as upper and lower dotteddashed lines, respectively. Lower panel: Evolution of the differential brightness temperature. Lines are the same as in the upper panel.See Ciardi \& Salvaterra (2007) for details.

with a mass of $300 \mathrm{M}_{\odot}$. In the upper panel we show the evolution of the spin temperature for a Salpeter (solid lines) and a VMS (dashed) IMF, and in the absence of a Ly $\alpha$ background (dotted). $T_{C M B}$ and $T_{k}$ are plotted as upper and lower dotted-dashed lines, respectively. In the lower panel the evolution of the corresponding differential brightness temperature is shown. At very high redshift $T_{s}=T_{k}=T_{C M B}$ and thus no signal is expected. Once the evolution of $T_{C M B}$ and $T_{k}$ decouples, collisions in the gas are efficient in maintaining an equilibrium between $T_{s}$ and $T_{k}$ and thus the line can be observed in absorption, but at $z<20$ collisions are not efficient anymore and a thermal equilibrium with the CMB is quickly reached. This is the epoch when the first sources of radiation turn on which, in addition to the ionizing photons that initiate the reionization process, emit also Ly $\alpha$ photons. If we assume that they are produced by a population of metal-free stars with either a Salpeter IMF or VMS we find that the $21 \mathrm{~cm}$ line can actually be observed also in this redshift range, initially in absorption against the $\mathrm{CMB}$, and, once the IGM has been heated above the $\mathrm{CMB}$, in emission. For the case shown in the Figure, the redshift at which the transition between absorption and emission takes place is $\sim 15$ (12) for a Salpeter IMF (VMS) (for details see Ciardi \& Salvaterra 2007).

In addition to Ly $\alpha$ photons, other sources of heating can be present in the high redshift uni- 

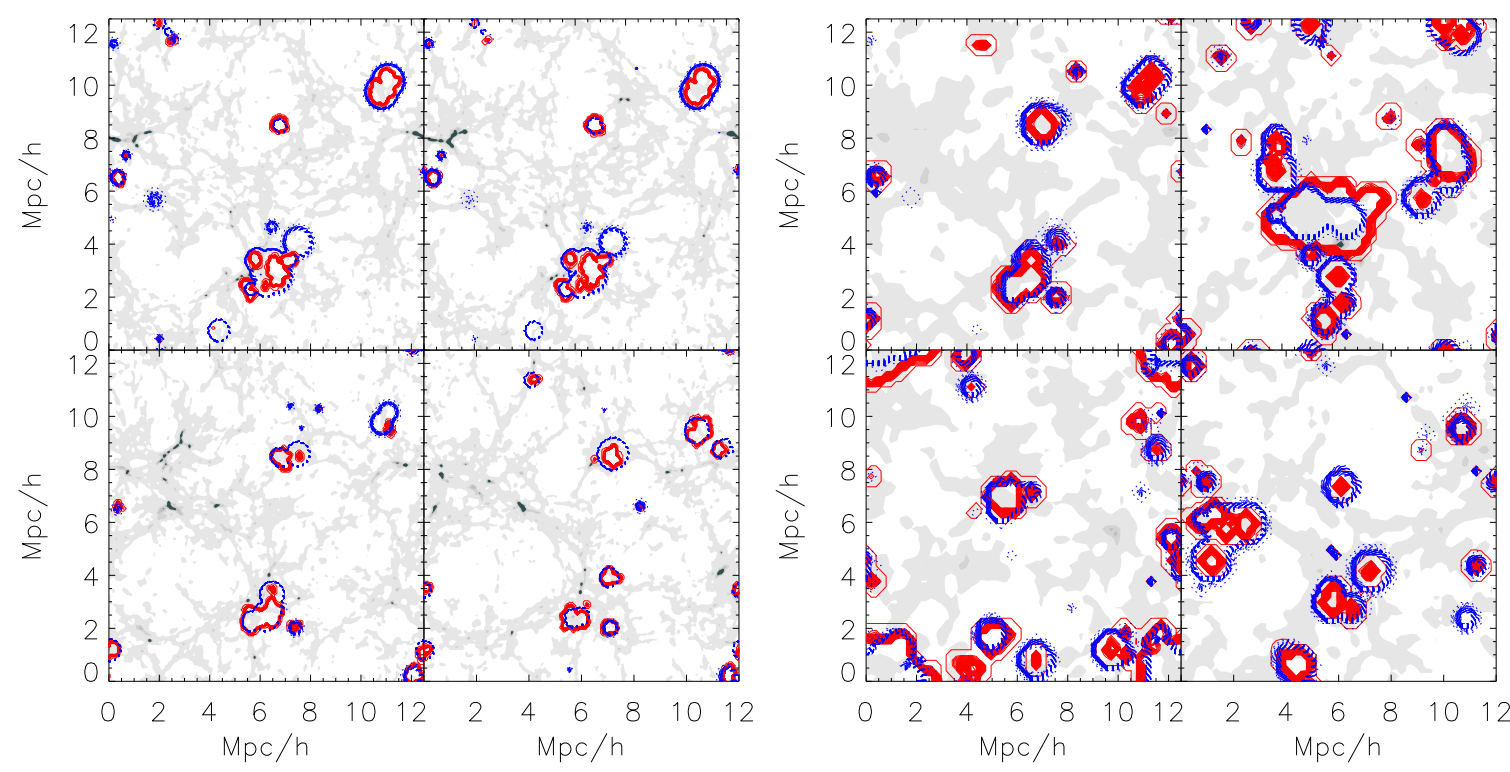

Figure 3: Four slices randomly selected along a direction in the box are plotted that displays the contours (three levels $[0,0.5,1]$ ) of the neutral fraction of CRASH (red) and BEARS (cyan) at $z=6$ in $256^{6}$ (left panel) and $64^{3}$ (right panel) grid box of $12 h^{-1} \mathrm{Mpc}$ comoving size. The underlying light gray contours represent the dark matter overdensities. See Thomas et al. (2009) for details.

verse, as e.g. x-ray photons from an early population of mini-quasars. Using the output of SPH simulations designed to follow the formation of massive black holes at $z \sim 6$ (Pelupessy, Di Matteo $\&$ Ciardi 2007), we have self-consistently estimated the relative contribution to heating from $x-$ rays produced by quasars and Ly $\alpha$ photons emitted by stellar type sources. We find that, for all the models considered, the x-ray heating is always dominant (Ciardi, Salvaterra \& Di Matteo 2009). Nevertheless, also in this case a homogeneous background has been assumed, while a proper radiative transfer of the various components should be followed to assess the exact contribution of UV, $\mathrm{x}$-rays and $\operatorname{Ly} \alpha$ photons to the ionization and heating of the gas.

Once the value of $T_{S}$ is known, from the distribution of $\mathrm{HI}$ provided by simulations of reionization maps of differential brightness temperature can be derived and analyzed to retrieve quantities relevant to observations. Ideally tomography of the IGM will be feasible, allowing to obtain maps of $21 \mathrm{~cm}$ emission/absorption at different redshift. In practice, this will not be achieved during the first few operational years of the present generation of radio telescopes (such as LOFAR or MWA) and instead statistical signatures (e.g. the power spectrum of fluctuations of differential brightness temperature) will be sought after.

\section{LOFAR}

Among others, the LOFAR telescope is already in a very advanced construction phase, with 39 stations already on the ground and 28 completed and integrated. The telescope is in its commissioning phase and data are flowing. Thus, ad hoc theoretical predictions should be available to be compared with observations. To this aim, very large boxes (several hundreds of $\mathrm{Mpc}$ ) are needed. On these scales the use of fully consistent 3D RT calculations (as the ones mentioned 


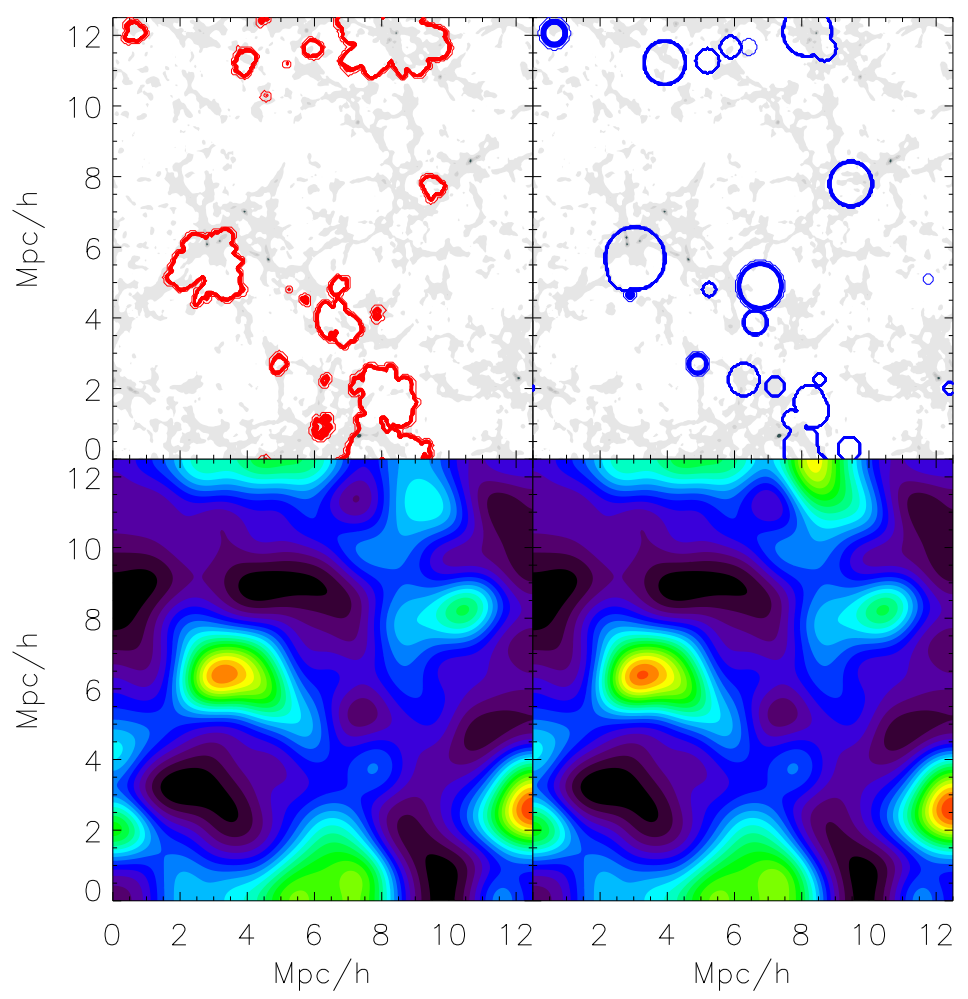

Figure 4: The ionization fronts of CRASH (red contours, top left) and BEARS (blue contours, top right) are overplotted on the underlying density field shown in light grey. This slice is extracted from a $256^{3}$ box at redshift six. The corresponding figures below show the images after being smoothed by the beam response of the antenna. See Thomas et al. (2009) for details.

above) is prohibitively expensive and an alternative approach is needed. Within the LOFAR Epoch of Reionization (EoR) Working Group a great effort in this direction is being made. In particular, a method has been developed based on a combination of numerical simulations and the 1D RT code BEAR (Thomas \& Zaroubi 2008). Before applying this method to produce realistic, very large scale reionization histories, its validity needs to be checked against a full RT approach. We have thus compared the performances of CRASH and BEAR on smaller boxes in a variety of test cases (Thomas et al. 2009). In Figure 3 random slices cut through the simulation boxes are shown for different grid sizes at $z=6$. The lines are isocontours of neutral fraction. Although the details of the reionization structure present some differences due to the inherent spherical symmetry of the 1D approach, the overall agreement is remarkably good, of the order of few percents in terms of average neutral fraction.

What is more important though is that, once the simulations have been convolved with the noise and the beam response of the telescope, the images look almost identical, as can be seen in Figure 4. This means that, for the purposes of modeling the reionization process for LOFAR observations the approximated approach is sufficient.

The final aim is to produce a series of reionization histories associated to the $21 \mathrm{~cm}$ signal. On top of these, the contribution from both extragalactic and Galactic foregrounds will be added to obtain mock observations as the example shown in Figure 5. The ultimate challenge for the 


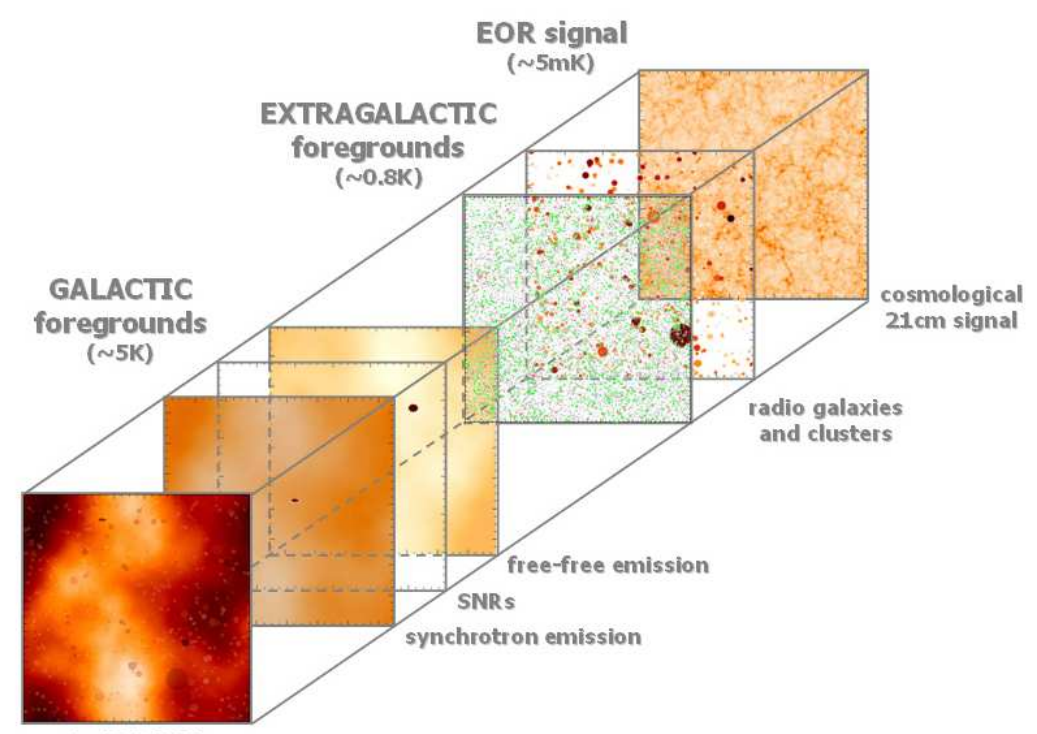

(@) $120 \mathrm{MHz}$

Figure 5: Pipeline showing the building of a mock observation at a nominal frequency of $120 \mathrm{MHz}$ : on top of the cosmological signal, the extragalactic and Galactic foreground contamination is added (Jelic et al. 2008).

detection of the cosmological signal will be to separate it from the foreground signal, which is orders of magnitude higher. Thus, in addition to simulating mock reionization histories an ongoing effort is being done to identify the best technique to extract the primary signal (e.g. Jelic et al. 2008; Harker et al. 2009a, 2009b).

\section{References}

[1] Bolton J. S., Haehnelt M. G., 2007, MNRAS, 374, 493

[2] Ciardi B., Ferrara A., Marri S., Raimondo G., 2001, MNRAS, 324, 381

[3] Ciardi B., Scannapieco E., Stoehr F., Ferrara A., Iliev I. T., Shapiro P., 2006, MNRAS, 366, 689

[4] Ciardi B., Ferrara A., White S.D.M., 2003, MNRAS, 344, L7

[5] Ciardi B., Salvaterra R., 2007, MNRAS, 381, 1137

[6] Ciardi B., Salvaterra R., Di Matteo T., 2009, MNRAS, in press, arXiv:0910.1547

[7] Ciardi B., Stoehr F., White S.D.M., 2003, MNRAS, 343, 110

[8] Dunkley et al. 2008, astro-ph/0803.0586

[9] Field G. B., 1959, ApJ, 129, 551

[10] Furlanetto S. R., Oh S. P., Briggs F. H., 2006, Phys. Rep., 433, 181

[11] Harker G., et al., 2009a, MNRAS, 393, 1449 
[12] Harker G., et al., 2009b, MNRAS, 397, 1138

[13] Hirata C. M., 2006, MNRAS, 367, 259

[14] Iliev I. T., et al., 2006, MNRAS, 371, 1057

[15] Jelic V., et al., 2008, MNRAS, 389, 131

[16] Kogut A., et al. 2003, ApJS, 148, 161

[17] Larson D., et al. 2010, arXiv1001.4635

[18] Lehnert M. D., et al. 2010, Nature, 467, 940

[19] Maselli A., Ciardi B., Kanekar A., 2009, MNRAS, 393, 171

[20] Maselli A., Ferrara A., Ciardi B., 2003, MNRAS, 345, 379

[21] Maselli A., Gallerani S., Ferrara A., Choudhury T. R., 2007, MNRAS, 376, L34

[22] Mesinger A., Haiman Z., 2007, ApJ, 660, 923

[23] Oh S. P., Furlanetto S. R., 2005, ApJ, 620, L9

[24] Ota K., et al., 2007, ApJ, 677, 12

[25] Ouchi M., et al., 2009, ApJ, 696, 1164

[26] Pelupessy I., Di Matteo T., Ciardi B., 2007, ApJ, 665, 107

[27] Pierleoni M., Maselli A., Ciardi B., 2009, MNRAS, 393, 872

[28] Pritchard J. R., Furlanetto S. R., 2006, MNRAS, 367, 1057

[29] Salvaterra R., et al., 2009, Nature, 461, 1258

[30] Spergel D. N., et al. 2007, ApJS, 170, 377

[31] Springel V., Yoshida N., White S. D. M., 2001, NewA, 6, 79

[32] Thomas R. M., et al. 2009, MNRAS, 393, 32

[33] Thomas R. M., Zaroubi S., 2008, MNRAS, 375, 1269

[34] Wouthuysen S. A., 1952, AJ, 57, 31

[35] Wyithe J. S. B., Loeb A., 2004, Nature, 427, 815 